\title{
Quantification of the direct solar impact on some components of the hydroclimatic system
}

\author{
Constantin Mares *, lleana Mares *, Venera Dobrica and Crisan Demetrescu \\ Institute of Geodynamics of the Romanian Academy, R-020032 Bucharest, Romania \\ *Correspondence: constantin_mares_ro@yahoo.com (C.M.); \\ ileana_mares@geodin.ro (I.M.)
}

\begin{abstract}
This study addresses the causal links between external factors and the main hydro-climatic variables. There is a gap in the literature on the description of a complete chain in addressing the structures of direct causal links of solar activity on the terrestrial variables. This is why, the present study uses the extensive facilities of the application of information theory in view of recent advances in different fields. Also, by other methods (e.g. neural networks) first are tested the existence non-linear links of solar-terrestrial influences on hydro-climate system. The results are promising related to the solar impact on terrestrial phenomena which is discriminant in spacetime domain. The implications prove robust for determining the causal measure of climate variables under direct solar impact which makes it easier to consider solar activity in climate models, by appropriate parametrizations.

This study found that hydro-climatic variables are sensitive to solar impact only for certain frequencies (periods) and these have a coherence with the Solar-Flux only for some lags of the Solar-Flux (in advance).
\end{abstract}

Keywords: time series; causality; entropy transfer; wavelet analysis; neural networks; climate response; solar impact.

\section{Introduction}

In the climate system the processes that take place are due to the combination of two main factors the solar external factor and its own internal mechanism. The complexity of this combination is difficult to quantify space-time by deterministicexplicit or stochastic-dynamic models [1, 2]. The relationship between the internal and external determinants factors in the evolution of the terrestrial climate system remains quite unknown, despite the recent and increasingly sophisticated modeling.

In general, different pros and cons arguments try bring light to different statistical methods or deterministic models about their suitability to the peculiarities of sun-climate connection.

There are also cases when it is not possible to adequately capture climate responses to solar forcing even through robust models coupled ocean-atmosphere of general circulation. Thus the sensitivity of some climate hydroclimatic processes may not represent true responses when a physical parameter does not respond linearly with the solar forcing [2] . In turn even hydroclimatic processes between them have 
nonlinear links [3]. These links were highlighted using joint entropy method. Smith [4], use mutual information (MI) to calculate nonlinearity and looks at $\mathrm{MI}$ as a measure of total dependence between random variables. Goodwell et al. [5] discusses the advantages and disadvantages of applying information theory to the links of different variables in earth sciences, trying to find a measure of these links.

Multiple more or less sophisticated methods of detecting Granger-type causality between different factors for different fields [6-8], have been developed. Others investigators developed some information measures via predefined functions, copula functions [9] or relative information generating function as defined by Guiasu and Reischer [10]), Hao and Singh [11]. Many applications attempt to aggregate important factors to determine the evolution of natural phenomena on Earth, but few succeed.

Why is that? Because the method of discerning the contribution of each factor as from cause to effect, is not the most appropriate. It is crucial that the method of discrimination can quantify the contribution of each factor from a lot of factors that contribute to the determination of a phenomenon, and the transfer of entropy (TE) offers this desire to the full [12-14]. In addition TE provides the adequate tool for relevant physical interpretations.

However, there happens to be errors of interpretation regarding TE itself [15], so this tool should be used with great care. Therefore, details that are not yet known about the physical mechanisms that govern terrestrial climate behavior remain to be found. Discrimination of the direct effects of solar activity on processes in the climate system is difficult. Solar disturbance, even quite strong, is modulated by the non-linearities existing between it and hydroclimatic factors [16].

In addition, even the internal mechanism of the atmosphere, it itself can produce notable climatic extremes [16-18]. As a secondary issue, the reassessment of the sun impact on climate is also necessary due to the fact that modern, satellite measurements outside the modulating space of the Earth's atmosphere are more correct $[19,20]$.

Is it enough to find the nonlinearities and elucidate the problem of the sunclimate link? The situation is so complex that we have to evaluate the structure of the causal chain both between geophysical factors and between geophysical factors and the sun. Depending on this situation, as well as the current state of knowledge, we decided to do the investigations to elucidate at least partially the existing problems.

In this study we first test the links between the external factor by the Solar Flux parameter $10.7 \mathrm{~cm}$ and the hydroclimatic variables, whether or not they are nonlinear by method proposed by [21,22]. If the links are nonlinear we test complexity by appropriate measures, provided to a large extent in the paper [23]. Entropy may be looked as a measure of complexity [24]. Of course, informational entropy in general and especially entropy transfer is regarded today as method in advanced sciences [25]. But entropy can be seen not only as a measure of complexity but also as a measure of causality more precisely the transfer of entropy, in the interrelationships of natural processes [26]. Palus and Vejmelka [27], Goodwell and Kumar [28], consider the conditional mutual information term is equivalent to the transfer entropy. It was also found that some source processes, recorded in hydrometeorological time series are more active processes compared to others counted over longer or shorter periods [5]. For other periods, the roles are changed between source and target [29], i.e. sources become passive (target) and target become sources (active).

The main objective of our investigation in this paper is to establish the causal chain of the solar impact with the respective weights, on the hydroclimatic processes 
using the theory of information, that is able to capture aspects, which deterministic methods by inherent constraints, cannot do. During our investigations, as we will see in the following, the results are not always significant. Significant signals appear on portions of the analyzed time series.

Therefore is important to find measures of process interaction in the time- frequency domain. We chose the situation of the aposterior knowledge of the dual manifestation in time-frequency of sun-climate processes through wavelet analysis [30]. How this has achieved will be seen in the paragraph below in which we mention the characteristic intervals and the consistency of the processes. It is not yet enough to know only qualitatively the details of the physical mechanisms that govern the behavior of the terrestrial climate, but also the weights of the solar impact.

Some investigations start from modeling the solar activity signal on the climate indices [31], knowing apriori partial characteristics. With the help of these models the authors come to the interesting conclusion that suggests a causal link between solar activity and pseudo-periodicities of most climate indices. The method, although essentially linear, applied to nonlinear structures, manages to capture a solar footprint on a significant part of the terrestrial phenomena recorded by climatic indices.

Important is to establish the existence of laws as from cause to effect, between external factors and the own mechanism of the geophysical environment considered by hydroclimatic indices. This is the approach we are trying to implement here by methods that implicitly capture the non-linearities of the considered links. We thus manage to discriminate between external causes and hydro-climatic factors. This is the first step towards proper modelling of hydroclimatic processes under the impact of the external factor.

From a theoretical point of view, things are quite advanced [26]. But from a application point of view, in some papers [5,32], were used estimates of the links of active causal factors (sources) and receptors (passive) in a linear manner through representations in the space of wave numbers such as the development in the Fourier series.

In general, the pros and cons different arguments there are for the some statistical methods, as to their suitability for the nature of the connection between phenomena. That is why it is good for us to first test the nature of the link between phenomena and then to do the investigations accordingly. As, in the domain of sun influence on the hydroclimatic system with focus on the Danube basin, there are few works that address the problem from cause to effect, we try here to elucidate some aspects of the direct solar impact by applying the theory of information a posterior.

\section{Data}

We consider the climatic variables that govern the synoptic scale through their seasonal averages. We did not consider the climate variables by our innate desire quoting [1] because, as demonstrated, the impact of the sun on the climate has distinct characteristics for the summer season compared to the winter season and even the transition seasons [33]. The present investigation refers to the seasonal, unfiltered time series from 1948 to 2000 . We therefore have a statistical volume of 53 values, sufficient for entropy transfer analysis [34]. 


\subsection{Regional Scale}

The Lower Danube Basin discharge was recorded by Orsova station (ORS_Q), located at the entrance of the Danube in Romania. It represents an integrator of the upper and middle basin. Data was provided by the National Institute of Hydrology and Water Management, Bucharest, Romania. For each station, of the 15 considered in the Danube basin, was estimated a simple drought index (TPPI), which is calculated by the difference between standardised temperatures and precipitation.

\subsection{Large scale}

In order to see the influence of large-scale atmospheric circulation on the variables at the regional scale, we considered the seasonal mean values of the sea level pressure field (SLP) in the sector $50^{\circ} \mathrm{W}-40^{\circ} \mathrm{E}, 30^{\circ}-65^{\circ} \mathrm{N}$. SLP data were available at http://rda.ucar.edu/datasets/ds010.1 of the National Center for Atmospheric Research (NCAR). The 5-degree latitude/longitude grids, computed from the daily grids, begin in 1899 and cover the Northern Hemisphere from $15^{\circ} \mathrm{N}$ to the North Pole. The North Atlantic Oscillation index (NAOI) was downloaded from http://www.Ideo.columbia.edu/res/pi/NAO/.

The Greenland-Balkan Oscillation index (GBOI), introduced by Mares et al. 2013 [35], was calculated using the correlative analysis of the first principal component (PC1) of the Empirical Orthogonal Functions (EOFs) for the precipitation field at the 15 stations of this study with sea level pressure (SLP) at each grid point where it was defined. Then GBOI is calculated as differences of normalized SLP at Nuuk and Novi Sad, located in opposite sign correlation nuclei [35].

NAO and GBO circulation indices can capture certain aspects of climate processes on a planetary and continental scale respectively.

Atmospheric blocking as a variable is one of the most important phenomena whose genesis cannot be reproduced by sophisticated deterministic models. The atmospheric blocking is important by its association with extreme hydroclimatic events and it is modulated by solar activity [36]. The calculation of blocking indices involves pressure differences between mid-and northern latitudes. The geopotential at $500 \mathrm{hPa}$ provided by British Atmospheric Data Centre (BADC) (https://badc.nerc.ac.uk/home/index.html).Three sectors were taken into account: Atlantic-European (AE) on the domain $\left(50^{\circ} \mathrm{W}-40^{\circ} \mathrm{E} ; 35^{\circ} \mathrm{N}-65^{\circ} \mathrm{N}\right)$, Atlantic (A) defined in $\left(50^{\circ} \mathrm{W}-0^{\circ}, 35^{\circ} \mathrm{N}-65^{\circ} \mathrm{N}\right)$ and European $(\mathrm{E})$ in the region $\left(0^{\circ}-40^{\circ} \mathrm{E} ; 35^{\circ} \mathrm{N}-\right.$ $\left.65^{\circ} \mathrm{N}\right)$. The corresponding blocking indices are $A E B I, A B I, E B I$, respectively.

Why did we choose the climatic variables at the level of the terrestrial surface or the average troposphere? For the simple fact that the sun-climate relationship, although evident in the higher atmospheric levels, at the the earth's surface is not evident by the earlier investigations $[33,37]$ and in newer ones it is partially clear $[36,38]$.

\subsection{Solar Flux index}

For the time interval 1948-2000, the solar forcing is quantified by the solar radio flux at $10.7 \mathrm{~cm}$ (usually called $\mathrm{F} 10.7$ index). Details on the $10.7 \mathrm{~cm}$ solar radio flux and its applications are given in [39].

Why did we consider solar activity through Solar Flux? For the simple fact that older and even newer studies have highlighted qualitative links between Solar Flux 10.7 with atmospheric variables like temperature at the isobaric level of $30 \mathrm{hPa}$ 
in the stratosphere [33]. Newer studies that have shown good representation of total solar radiation (TSI) by Solar Flux $10.7 \mathrm{~cm}$ which recommends it to be considered both in deterministic models as well as stochastic ones [40].

The Sun's external factor's impact on hydroclimatic processes at the ground surface suffers significant modulations [41]. These modulations are due to the internal mechanisms of the components of the climate system then explained in more detail by [42] by supposition the existence of a stratospheric jet which interacts with the atmospheric waves. These waves then transfer energy throughout the troposphere [43]. The role of solar activity in this transfer would be to increase the conversion of the baroclinic energy of the current jet to eddies with heat exchange to eddies.

\section{Methods}

\subsection{Preliminaries}

It is very important to determine whether the link between the variables describing the phenomena in question is linear or nonlinear. This analysis is carried out by appropriate methods of neural networks [21,44]. For example, the link between the Solar Flux and the NAOI, as well as the Danube discharge at the Orsova station, by applying neural network models to the analysis of nonlinear canonical correlation, following Hsieh and Tang [21], Hsieh [22] we found that it is clearly nonlinear.

The difference in timing is crucial in considering mechanisms to explain solar-climate links [45] and the non-linearity link test should also be applied in this case. But more important is how we must take this difference in timing. And this is one of the purposes of this study. We are looking for such a lag in the time-frequency domain by the wavelet transform [30] so that there is coherence between the Solar Flux and the considered hydroclimatic variables (Figure 2). In this way we establish exactly the the lag between the Solar Flux (in advance) and the terrestrial variables. 
(a)

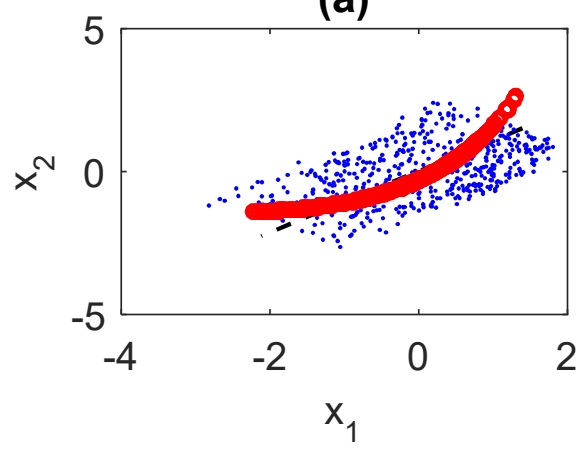

(c)

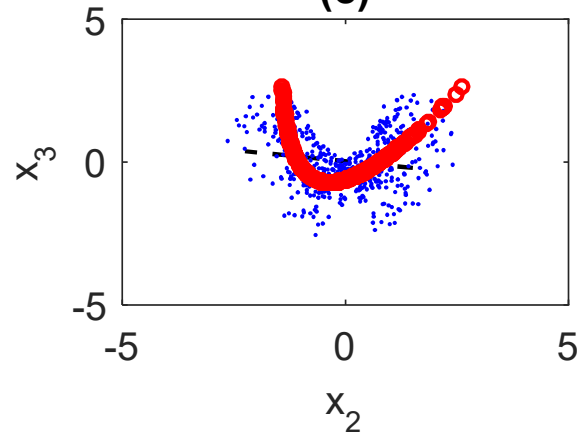

(b)

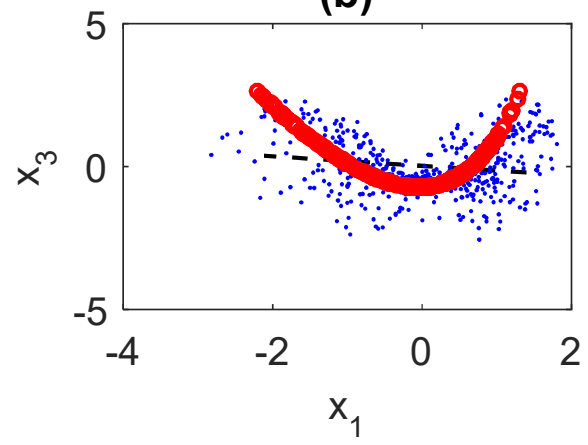

(d)

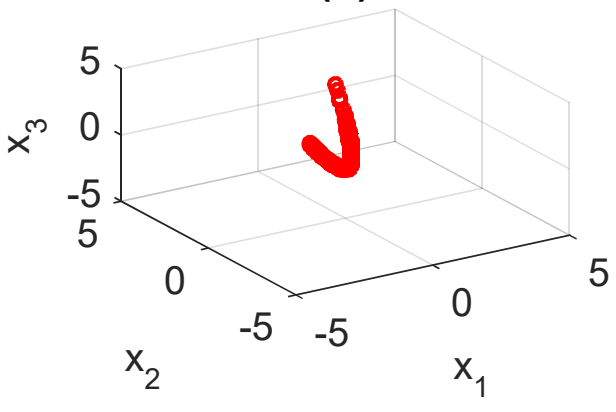

Figure 1. Non-linearities between the Solar Flux (X1) and the climate variables NAOI $(X 2)$ and the Danube discharge at the Orsova station (X3), during the winter (19482000). The plane projection: (a) for (X1,X2); (b) for (X1,X3); (c) for (X2,X3) and (d) space projection for $(\mathrm{X} 1, \mathrm{X} 2, \mathrm{X} 3)$

\subsection{The information theory elements}

Applying information theory to essentially dynamic systems brings us more information in explaining what governs geophysical processes under the impact of solar external forcing. It is it is understandable that solar activity under its various forms should lead the phenomena of the earth. But also between the various hydroclimatic phenomena we deal with there must be a causal link. A huge leap was made from Granger's simplest causal link [46] to the robust nonlinear type based on informational entropy defined by Schreiber [26].

Mutual information (MI) is defined by

$$
M I(X, Y)=H(X)+H(Y)-H(X, Y)
$$

$\boldsymbol{H}$ - information entropy of the variables $\boldsymbol{X}$ and $\boldsymbol{Y}$ [47])

As is shown in [48] a method for quantifying the transfer of information from one variable to another was developed by [26] and was then applied in many investigations $[28,49,50]$. 
According to Timme and Lapish [51] transfer entropy, using conditional mutual information is given by [26]

$$
T E(X \rightarrow Y)=M I\left(Y_{\text {future }} ; X_{\text {past }} / Y_{\text {past }}\right)
$$

In this study we used Timme and Lapish's MATLAB calculation routines [51].

Apart from using MI to estimate TE (equation 2), this measure is used in different applications such as calculating the nonlinear correlation coefficient. Thus in [52] nonlinear connections between the five atmospheric predictors and the Danube discharge were tested using the nonlinear correlation coefficient (NLR) based on mutual information. Significant connections between the predictor and the predictand indicated a nonlinear link between them, the NLR being generally higher than the linear correlation coefficient.

\section{Results}

The results shown in the Figure 2 (top panel), highlight the wavelet coherence of two phenomena (Solar Flux and NAOI) in winter. The left-right arrows indicate phase difference $(\mathrm{df}=0)$ at least for a frequency spectrum corresponding to periods of 8-12 years. In Figure 2 (bottom panel) is presented the evolution of Solar - Flux and NAOI for the period 1948-2000. In the both graphical representation, the flux is taken with 3 years before the NAOI.
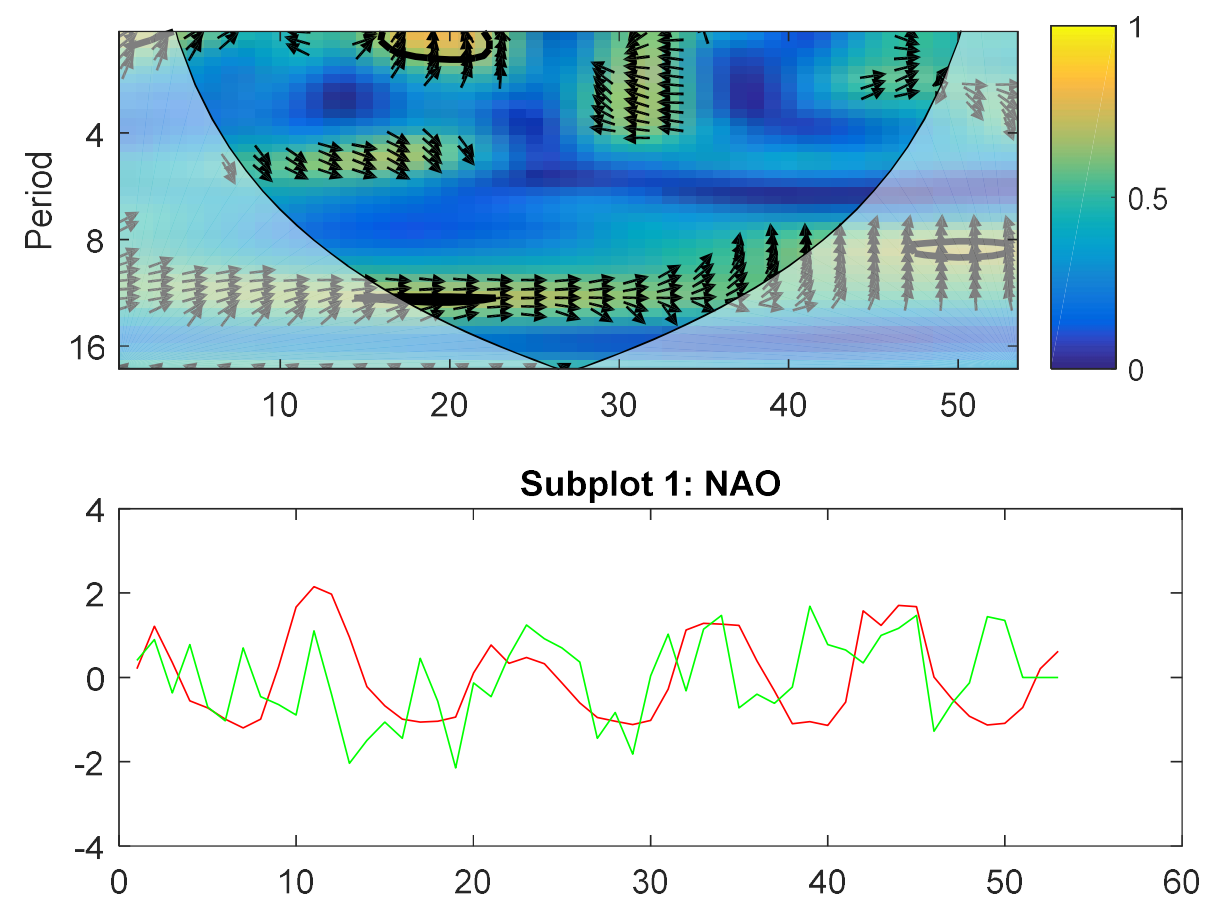

Figure 2. Top panel: Wavelet coherence of between Solar Flux and NAOI (above panel). Bottom panel: Evolution of the Solar - Flux (red) and NAOI (green) for winter season (1948-2000). The flux is taken with 3 years before the NAOI. 
It can be seen at the top of Figure 2, a good coherence (left-right horizontal arrows) for periods between 8-12 years for the first 35 years. The corresponding amplitudes of the solar (red) and NAO (green) are in concordance in the first 35-40 years (the bottom of the Figure 2). This is the role of the wavelet analysis to establish coherence at frequency-time domain.

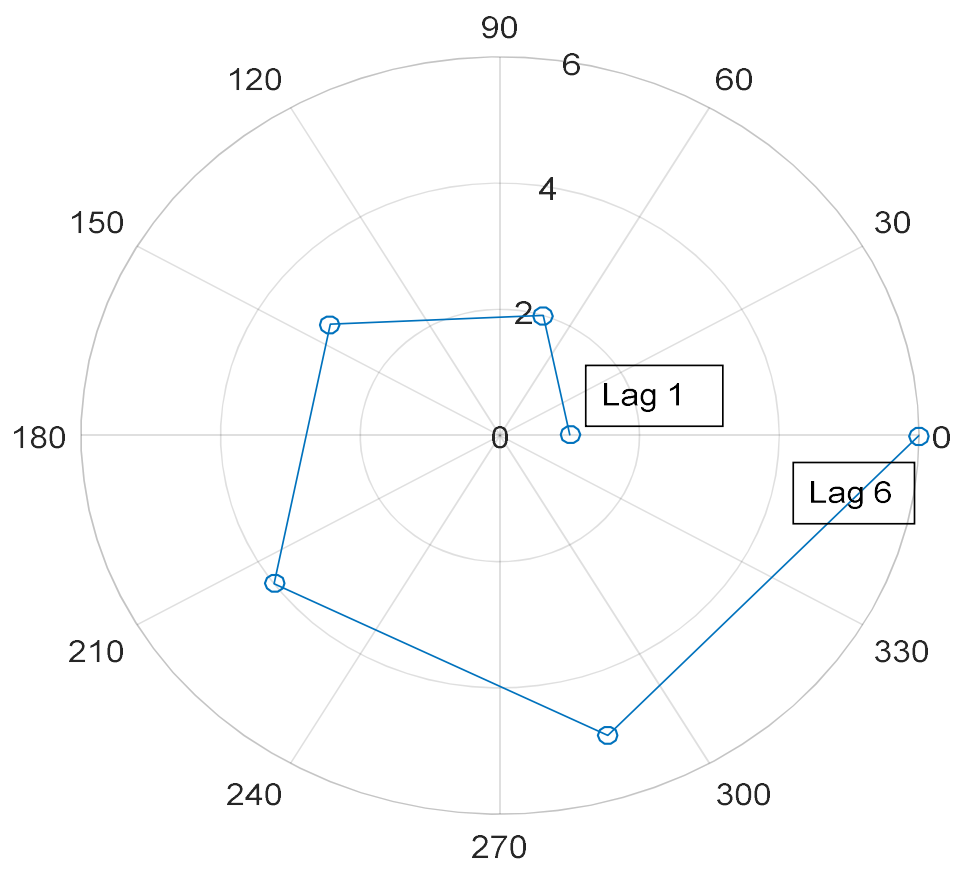

Figure 3. Representation the lags between the Solar - Flux (in advance) and NAOI, from lag- 1 to lag- 6 in winter time.

This graphic is obtained by calculating wavelet coherence [30] for different lags. Lag 6 represents the moment when $\mathrm{df}=0$ is reached again, ie the two phenomena are in phase. And for other terrestrial variables we found that they are sensitive to solar impact only for certain frequencies (periods) and these have a coherence with the Solar Flux only for certain lags of the Solar Flux (in advance).

In the following, a bidirectional analysis of the entropy transfer is made and the results are presented in Figures 4-7. The results are obtained by the procedure described in [51], and we have chosen the level of statistical significance with $p<0.1$. More recently the statistical significance is also achieved through other procedures [53].

In the Figures 4-7, for each season, the transfer entropy from Solar-Flux to seven terrestrial variables, are presented, but only for cases where TE is statistically significant. The test was achieved with solar indice taken with the lag from 1 to 5 years before terrestrial variables. In the center of figure is the source (Solar- Flux) from which the arrows start to the variables "target" or receiver. The arrow shows for each variables, value of TE and the significance level (p).

We notice that in the spring season (Figure 4 ) there are four situations with entropy transfer with statistical significance of $p<0.1$ respectively for $A E B I, A B I$, GBOI and TPPI, for which it can be said that the Solar- Flux has a direct influence on the climatic phenomena described by the respective indices. For the summer season 
(Figure 5) only one indice has specified statistical significance, namely $A B I$. In the fall (Figure 6) it is observed that the significant causal solar impact is on EBI and NAO. In winter (Figure 7) besides AEBI appears as significant, the discharge of the Danube to Orsova. Therefore, if we refer only to discharge, the direct causal impact on the Danube discharge is evident only in the winter season with a delay of 3 years.

It should be noted that here we chose the delay of the series in such a way as to keep the statistical volume of 53 years constant. This is done by replacing the values that are set off in series by zero. The error is not too large up to a lag $=5$, since the data is standardized. The scale on each figure has no meaning, but is important only for editing figure.

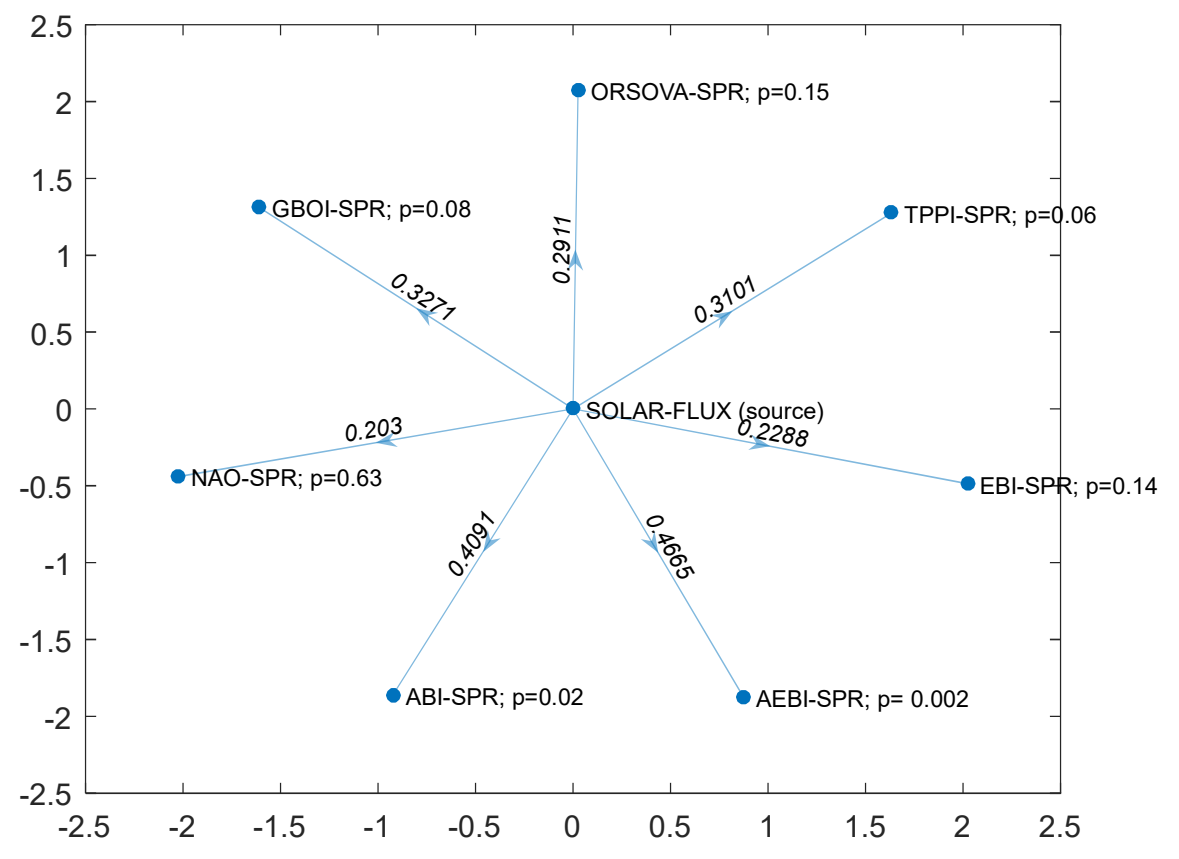

Figure 4. Distribution of entropy transfer (direct influence): source (Flux), receiver (variables mentioned) with the specific lag of maximum coherence for the spring season ( $p<0.1$ is significant) and the value TE inscribed on the arrow. High statistical significance, have only variables with corresponding lags: GBOI with lag=1; $A B I$ with lag=4; $A E B I$ with lag=1 and TPPI with lag=2. 


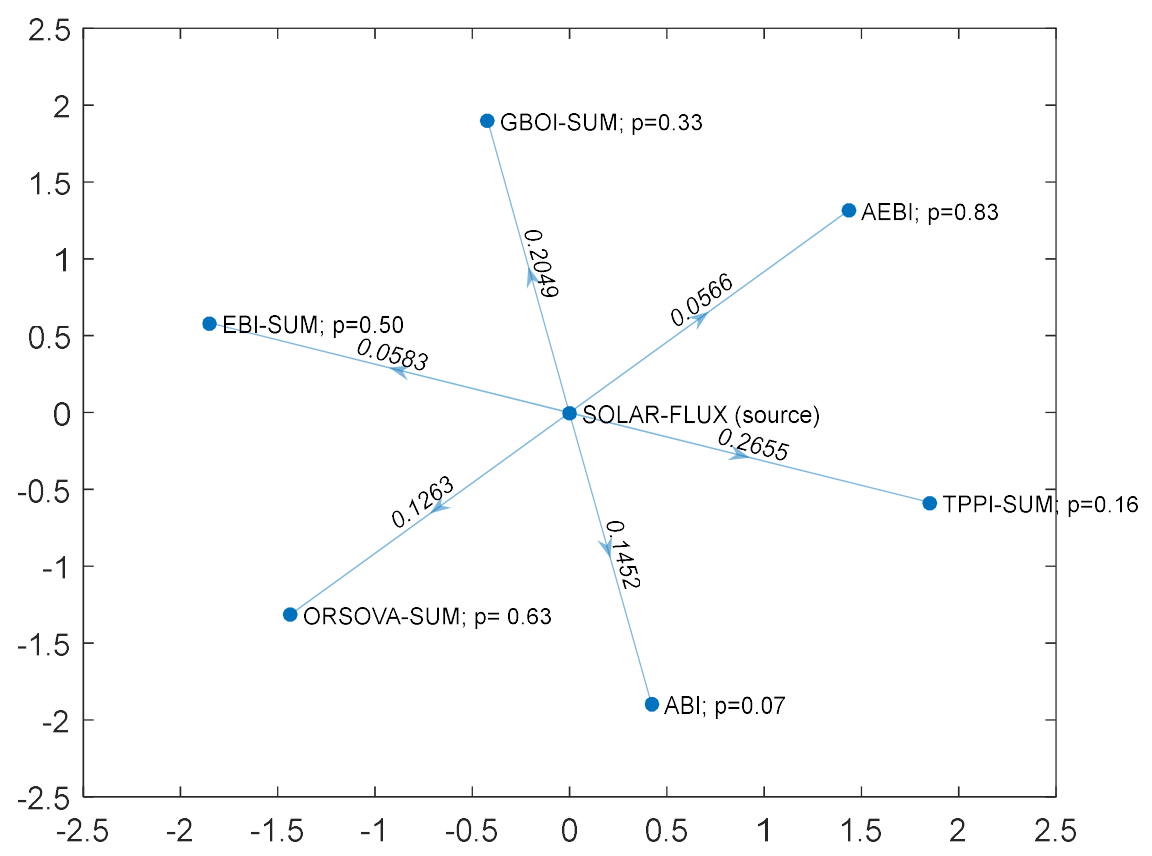

Figure 5. Same as Figure 4 but for summer, with significant $p$ only for $A B I \quad(\operatorname{lag}=5)$.

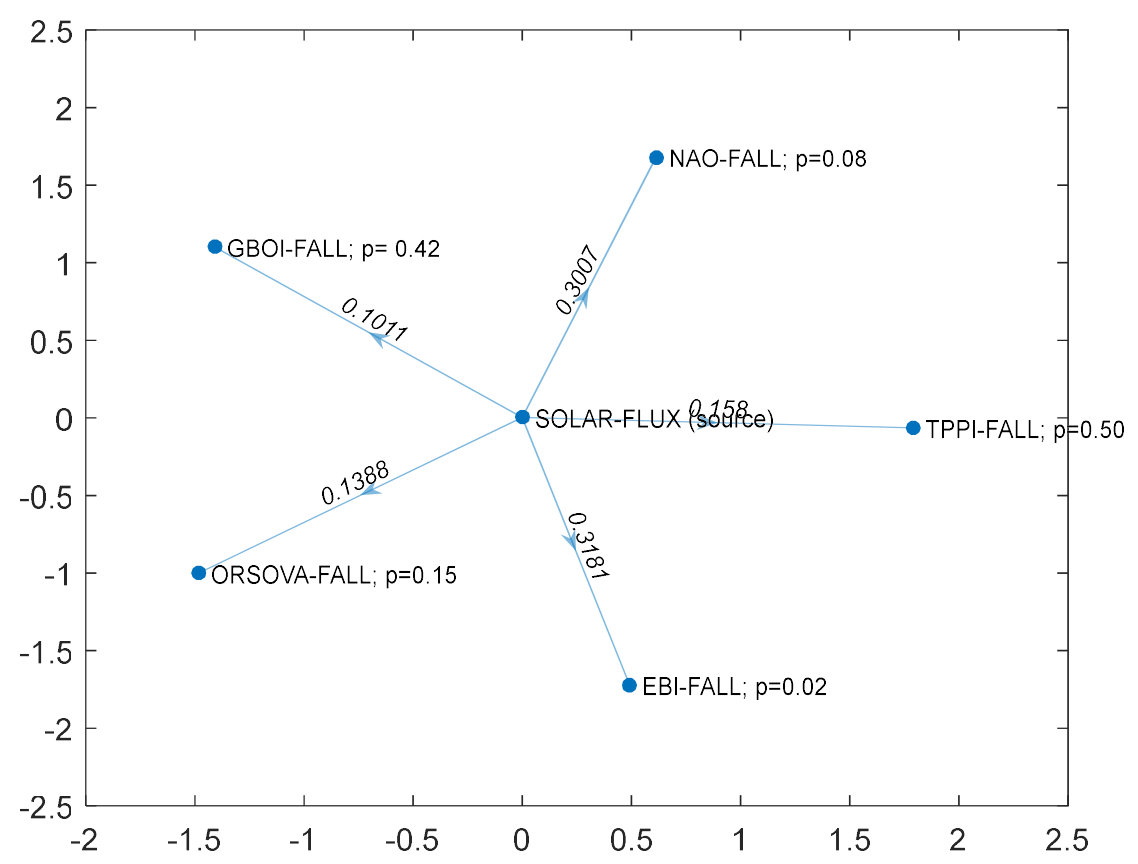

Figure 6. The same as Figure 4, but for autumn and with with statistical significance: $\mathrm{NAO}$ with lag=4 and $\mathrm{EBI}$ with lag=4. 


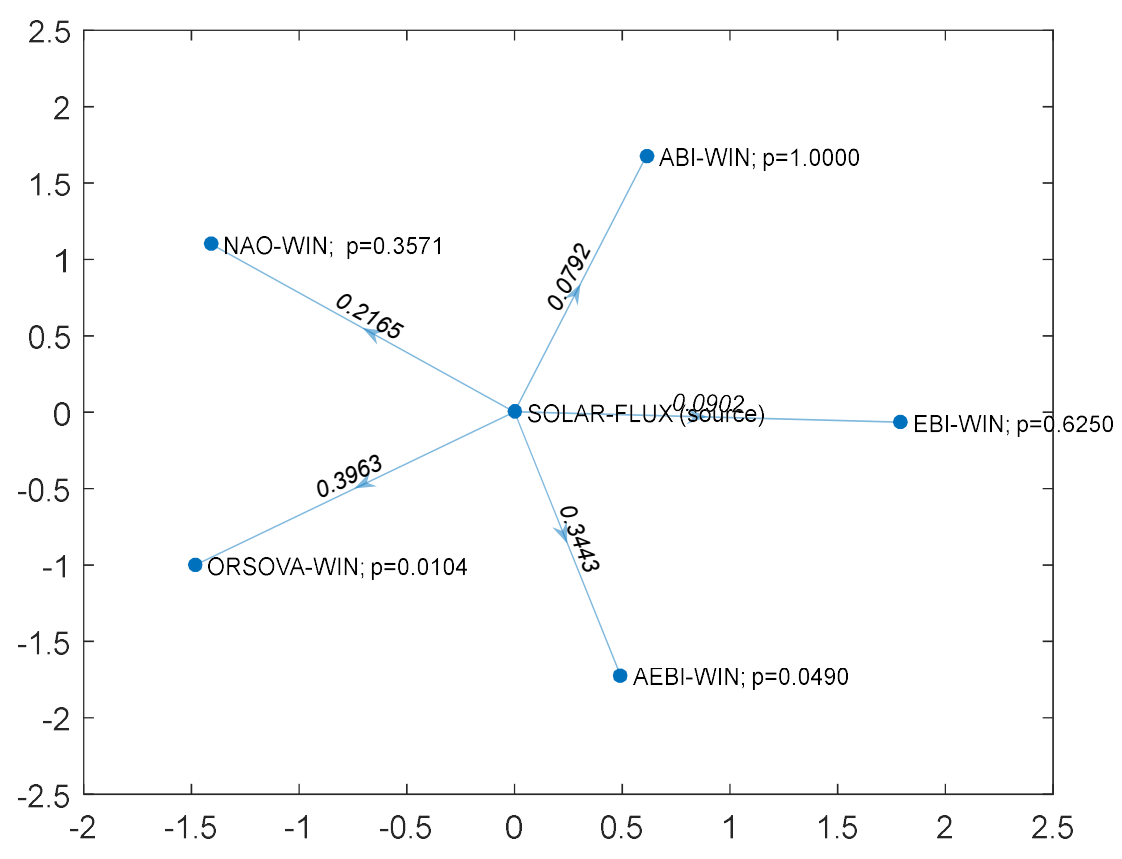

Figure 7. The same as Figure 4, but for winter, with significant variables, AEBI with lag $=5$ and ORS with lag=3.

The variables that do not appear in the figures are interpreted as not receiving direct influence. In the theoretic work [54] valuable by specifying the scope of validity of certain measures (MI), the hypothesis regarding the detection of coupling by transfer of entropy (or any other measure) namely that "it is valid only when the oscillators are not synchronized" does not come true. However, in practice there are spectral windows of coupled oscillators through which TE has detection value in two-way processes, of situations of causal determination.

\section{Conclusions and FurtherWork}

In this study it was found that hydro-climatic variables are sensitive to solar impact only for certain frequencies (periods) and these have a coherence with the Solar- Flux only for certain lags of the Solar- Flux (in advance).

The Danube discharge in the lower basin at Orsova station is directly sensitive (after a time interval of $\sim 3$ years) to the solar activity only in the winter season. In the transition seasons (spring, autumn) as well as in summer, the discharge of the Danube in the lower basin, of course, besides the solar activity, is also caused by other factors that determine the evolution of the discharge.

The conclusion is that we can talk about a causal dependence of those phenomena with positive TE with a specific "delay" and the interpretation of these relationships is much relieved. The selection of these causal dependencies open the way to adequate modeling and conforming to the evolution of hydro-climatic phenomena with discriminated solar impact. 
After establishing the nature of the connection between solar and considered geophysical variables, it is of interest the meaning of the link as from cause to effect. Detection of the type of causal connection between natural phenomena is of great interest both for objective explanation of mechanisms and for subsequent modeling. Of course, and other interesting ways of approaching such as described in $[31,55]$ to illustrate that nonlinear empirical modeling can help to disentangle complex climate interactions and various factors perhaps even external. The disadvantage of these methods whose robustness is obvious, is that must there are a prior knowledge about the processes that take place in each system.

The original contribution of this study is that the solar Flux 10.7 signal has a signature on the hydroclimatic factors focused on the Danube basin and that the impact of this signal is modulated by the internal mechanism of the atmosphere. Then, the way of obtaining these direct links as from cause to effect, is achieved by the robust method of entropy transfer applied after preliminary analysis. Preliminary analysis consists in optimizing the connection in the time-frequency domain with a appropriate lag to have a consistent coherence between the Solar Flux and each of the hydroclimatic variables.

The minus of this work stems from the fact that in the analysis carried out we postulated the existence of a single Solar Flux source that acts on a target. A crucial yet completely unresolved problem is that multi-source interactions lead to a possible redundant impact. Important steps to elucidate this problem were made by Lizier et al. [56].

This paper is only a short contribution on how to apply the robust method of information theory to the complex links of respective geophysical phenomena under direct solar impact.

Necessarily the next investigation that is required is to establish the links between the hydroclimatic variables themselves, in order to establish to what extent they are sources (active) or target (passive) and for how long they have these interchangeable characteristics.

The results of the investigation presented [29] it gives us hope that we are on the right way. We intend to apply this new kind of investigation to other problems in climate science.

Author Contributions: Conceptualization, Mares Constantin and Mares lleana; Formal analysis, Demetrescu Crisan; Investigation, Mares Constantin, Mares lleana, Dobrica Venera and Demetrescu Crisan; Methodology, Mares Constantin and Mares lleana; Resources, Dobrica Venera; Supervision, Demetrescu Crisan; Validation, Dobrica Venera; Writing - original draft, Mares Constantin and Mares lleana.

Funding: This research received no external funding.

Conflict of Interest: The authors declare no conflict of interest.

\section{References}

1. Saltzman, B. A survey of statistical-dynamical models of the terrestrial climate. Advances in Geophysics. Academic Press, USA, New York San Francisco London, 1978, 20,183-304. 
2. Wen, G.; Cahalan, R. F.; Rind, D.; Jonas, J.; Pilewskie, P.; Dong L. Wu, D. L.; Krivova, N. A. Climate responses to SATIRE and SIM-based spectral solar forcing in a 3D atmosphere-ocean coupled GCM. Published by EDP Sciences, J. Space Weather Space Clim. 2017, 7, A11 DOI: 10.1051/swsc/2017009.

3. Zhang, G.; Su, X.; Singh, V. P.; Ayantobo, O. O. Modeling NDVI Using Joint Entropy Method Considering Hydro-Meteorological Driving Factors in the Middle Reaches of Hei River Basin. Entropy 2017,19, 502; doi:10.3390/e19090502.

4. Smith, R. A Mutual Information Approach to Calculating Nonlinearity. Stat. 2015, 4.1, 291-303. Doi:10.1002/sta4.96

5. Goodwell, A.; Jiang, P.; Ruddell, B.; Kumar, P. Debates -Does Information Theory provide a new paradigm for Earth science? Identifying causality, interaction, and feedback.WRR, 2020, 56, DOI: 10.1029/2019WR024940.

6. Seth, A. K. A MATLAB toolbox for Granger causal connectivity analysis. J. Neurosci. Methods 2010, 186, 262-273.

7. Barnett, L.; Seth, A. K. Behaviour of Granger causality under_Itering: Theoretical invariance and practical application. J. Neurosci. Methods 2011, 201(2),404-419.

8. Bressler, S. L.; Seth, A. K. Wiener-Granger causality: A well established methodology. Neurolmage 2011, 58, 323-329.

9. Aghakouchak, A. Entropy-copula in hydrology and climatology. Journ. of Hydromet. 2014,15(6), 2176-2189.

10. Guiasu, S. ; Reischer, C. The relative information generating function. Information Sciences. 1985, 35, 235-241.

11. Hao, Z.; Singh, V. P. Entropy-copula method for single-site monthly streamflow simulation. Water Resour. Res. 2012, 48, W06604, doi:10.1029/2011WR011419.

12. Manshour, P.; Balasis, G.; Consolini, G.; Papadimitriou, C.; Paluš, M. Causality and Information Transfer Between the SolarWind and the MagnetosphereIonosphere System. Entropy 2021, 23, 390.

https://doi.org/10.3390/e23040390.

13. Stumpo, M. ; Consolini, G.; Alberti, T; Quattrociocchi, V. Measuring Information Coupling between the Solar Wind and the Magnetosphere-lonosphere System. Entropy 2020, 22, 276; doi:10.3390/e22030276.

14. Pothapakula, P. K.; Primo, C.; Ahrens, B. Quantification of Information Exchange in Idealized and Climate System Applications. Entropy 2019, 21, 1094; doi:10.3390/e21111094.

15. James, R. G.; Barnett, N.; Crutchfield, J. P. Information Flows? A Critique of Transfer Entropies. Phys. Rev. Lett. 2016, Jun 10; 116(23):238701. doi: 10.1103/PhysRevLett.116.238701.

16. Hunt, B. G. Nonlinear Influences-A Kay to Short-Term Climatic Perturbations. J. A. S. 1988, 45, 387-395.

17. Lorenz, E. N. Nondeterministic Theories of Climatic Change. Quart. Res. 1976, $6,495-506$.

18. Ghil, M. A century of nonlinearity in the geosciences. Earth and Space Science 2019, 6, 1007-1042. https://doi.org/10.1029/2019EA000599.

19. Haigh, J. D.; Winning, A. R.; Toumi, R.; Harder, J. W. An influence of solar spectral variations on radiative forcing of climate. Nature 2010, 467, 696-699. 
20. Haigh, J. Solar influences on climate. Grantham Institute for Climate Change, 2011, Briefing Paper No. 5, Publisher: Imperial College London.

21. Hsieh, W. W.; Tang, B. Applying neural network models to prediction and data analysis in meteorology and oceanography. Bull. of the Amer. Meteor. Soc. 1998, 79(9), 1855-1870.

22. Hsieh, W. W. Nonlinear multivariate and time series analysis by neural network methods. Rev. Geophys. 2004, 42, RG1003, doi:10.1029/2002RG000112

23. Siggiridou, E.; Koutlis, C.; Tsimpiris, A.; Kugiumtzis, D. Evaluation of Granger Causality Measures for Constructing Networks from Multivariate Time Series. Entropy 2019, 21, 1080; doi:10.3390/e21111080.

24. Guiasu, S. Information Theory with applications. McGraw-Hill Inc., Great Britain, 1977.

25. Runge, J.; Nowack,P; Kretschmer, M.; Flaxman,S.; Sejdinovic, D. Detecting and quantifying causal associations in large nonlinear time series datasets. Sci. Adv. 2019, 5.11: eaau4996.

26. Schreiber, T. Measuring Information Transfer. Phys. Rev. Let. 2000, 85(2), 461- 464.

27. Palus, M.; Vejmelka, M. Directionality from coupling between bivariate time series: how to avoid false causalities and missed connections. Phys. Rev. 2007, E 75, 056211.

28. Goodwell, A.; Kumar, P. Information theoretic measures to infer feedback dynamics in coupled logistic networks. Entropy 2015, 17(11), 7468-7492.

29. Mares, I.; Mares, C,; Dobrica, V.; Demetrescu, C. Applications of the information entropy to quantify non-linear relationship between the precipitation in the Danube basin and the climate indices. EGU General Assembly Conference Abstracts, 2019, 21, 7115.

30. Grinsted, A.; Moore, J. C.; Jevrejeva, S. Application of the cross wavelet transform and wavelet coherence to geophysical time series. Nonlinear Processes Geophys. 2004, 11, 561-566.

31. Le Mouël, J. L.; Lopes; F.; Courtillot, V. A solar signature in many climate indices. J. of Geophys. Res.: Atmospheres. 2019, 124, 2600-2619.

32. Kumar, P.; Gupta, H. V. Debates-Does Information Theory Provide a New Paradigm for Earth Science?. Wat. Resour. Res. 2020, 56 (2), e2019WR026398.

33. Peixoto, J. P.; Oort, A. H. Physics of Climate. American Institute of Physics, 1992, $520 \mathrm{pp}$.

34.Ramos, A.M.T.; Macau, E.E.N. Minimum Sample Size for Reliable Causal Inference Using Transfer Entropy. Entropy 2017,19, 150.

35. Mares, I.; Mares, C.; Mihailescu, M. Stochastic modeling of theconnection between sea level pressure and discharge in the Danube lower basin by means of Hidden Markov Model. EGU General Assembly Conference Abstracts. 2013 15, 7606.

36. Barriopedro, D.; García-Herrera, R.; Huth, R. Solar modulation of Northern Hemisphere winter blocking. J. Geophys. Res. 2008, 113, D14118, doi:10.1029/2008JD009789.

37. McCormac, B. M.; Seliga, T. A. Solar-Terrestrial influences on weather and climate. In Proceedings of a Symposium/Workshop Held at the Fawcett 
Center for Tomorrow, The Ohio State University, Columbus, Ohio, 24-28 August; 1978.

38. Gray, L.J.; Ball, W.; Misios, S. Solar influences on climate over the Atlantic / European sector.In AIP Conference Proceedings 1810, 020002, 2017;

39. Tapping, K. F. The $10.7 \mathrm{~cm}$ solar radio flux (F10. 7). Space Weather 2013, 11(7), 394-406.

40.Bednarz, E. M.; Maycock, A. C.; Telford, P. J.; Braesicke, P.; Abraham, N. L.; Pyle, J.A. Simulating the atmospheric response to the 11-year solar cycle forcing with the UM-UKCA model: the role of detection method and natural variability. Atmos. Chem. Phys. 2019,19, 5209-5233.

41. Gossard, E.; Hooke, W. Waves in the Atmosphere.1975, pp. 456.

42. Kuroda, Y.; Kodera, K. Effect of solar activity on the Polar-night jet oscillation in the northern and southern hemisphere winter. Journ. of the Met. Soc. of Japan. 2002, 80, 973-984, 10.2151/jmsj.80.973.

43. Kuroda, Y. On the origin of the solar cycle modulation of the Southern Annular Mode. Journal of Geophysical Research: Atmospheres. 2018, 123, 1959-1969. https://doi.org/10.1002/2017JD027091.

44. Scholz, M.; Kaplan, F.; Guy, C. L.; Kopka, J.; Selbig, J. Non-linear PCA: a missing data approach. Bioinformatics 2005, 21(20), 3887-3895.

45. Roy, I.; Haigh, J. D. Solar cycle signals in sea level pressure and sea surface temperature. Atmos. Chem. Phys. 2010, 100, 3147-3153.

46. Granger, C.W. J. Econometrica. J. Econom. Soc. 1969, 37, 424.

47. Shannon, C. E. A mathematical theory of communication. Bell System Technical J. 1948, 27, 379-423.

48. Bennett, A.; Nijssen, B.; Ou, G.; Clark, M.; Nearing, G. Quantifying process connectivity with transfer entropy in hydrologic models. Wat. Resour. Res. 2019, 55, 4613-4629. https://doi.org/10.1029/2018WR024555.

49. Ruddell, B. L.; Kumar, P. Ecohydrologic process networks: 1. Identification. Wat. Resour. Res. 2009, 45, (3).

50. Hlinka, J.; Hartman, D.; Vejmelka, M.; Runge, J.; Marwan,N.; Kurths,J.; Palus, M. Reliability of Inference of Directed Climate Networks Using Conditional Mutual Information. Entropy 2013, 15, 2023-2045.

51. Timme, N. M.; Lapish, C. A. Tutorial for Information Theory in Neuroscience. eNeuro 2018, 5.3 0052-18.

52. Mares, I.; Mares, C.; Dobrica, V.; Demetrescu, C. Comparative study of statistical methods to identify a predictor for discharge at Orsova in the Lower Danube Basin. Hydrol. Sci. J. 2020, 65, 371-386.

53. Diks, C.; Fang, H. A Consistent Nonparametric Test for Granger Non-Causality Based on the Transfer Entropy. Entropy 2020, 22, 1123.

54. Sun, J.; Bollt, E. M. Causation entropy identifies indirect influences, dominance of neighbors and anticipatory couplings. Physica D: Nonlinear Phenomena 2014, 267, 49-57.

55. Timmermann, A.; Voss, H. U.; Pasmanter, R. Empirical dynamical system modeling of ENSO using nonlinear inverse techniques. J. Phys. Oceanogr. 2001, 31, 1579-1598.

56. Lizier, J.T.; Bertschinger, N.; Jost, J.; Wibral, M. Information Decomposition of Target Effects from Multi-Source Interactions: Perspectives on Previous, Current and Future Work. Entropy 2018, 20, 307. 